\section{Diurnal intraocular pressure reduction with latanoprost $0.005 \%$ compared to timolol maleate $0.5 \%$ as monotherapy in subjects with exfoliation glaucoma}

\author{
Abstract \\ Aims To compare the diurnal intraocular \\ pressure (IOP) efficacy and safety of timolol vs \\ latanoprost in subjects with exfoliation \\ glaucoma (XFG). \\ Methods A 3-month prospective, single- \\ masked, active-controlled, parallel comparison \\ performed in six centres in Greece that \\ randomized subjects in a 1:1 ratio to either \\ latanoprost in the evening (2000 hours) and \\ placebo in the morning ( 0800 hours), or timolol \\ twice daily (0800 and 2000 hours). \\ Results In all, 103 subjects completed the \\ study. After 3 months of chronic dosing, the \\ latanoprost group exhibited a trend to a \\ greater diurnal IOP reduction from an \\ untreated baseline $(24.9 \pm 3.2-17.4 \pm 2.9)$ \\ compared with timolol $(24.7 \pm 2.8-$ \\ $18.3 \pm 1.9 \mathrm{mmHg})(P=0.07)$. Latanoprost \\ showed a significantly greater IOP reduction \\ at 0800 hours $(-8.5$ vs $-6.0 \mathrm{~mm} \mathrm{Hg}$ for timolol, \\ $P<0.0001)$ whereas no difference was \\ observed between the two medications at 1000 , \\ 1400, and 2000 hours after a Bonferroni \\ Correction. In addition, latanoprost \\ demonstrated a narrower range of diurnal IOP \\ (2.4) than timolol $(3.2 \mathrm{mmHg})(P=0.0017)$. \\ Safety was similar between groups, except \\ there was more conjunctival hyperaemia with \\ latanoprost $(n=8)$ than timolol \\ $(n=1)(P=0.01)$. \\ Conclusions This study suggests that \\ latanoprost provides a statistically lower
}

AGP Konstas', N Mylopoulos², CH Karabatsas ${ }^{3}$, VP Kozobolis" ${ }^{4}$ S Diafas ${ }^{5}$, P Papapanos ${ }^{6}$, N Georgiadis ${ }^{1}$ and WC Stewart ${ }^{7,8}$

08:00-hour IOP and better range of IOP than timolol in the treatment of XFG glaucoma. Eye (2004) 18, 893-899. doi:10.1038/sj.eye.6701345 Published online 27 February 2004

Keywords: latanoprost; timolol; exfoliation glaucoma

\section{Introduction}

Exfoliation syndrome (XFS) is an age-related generalized disorder of the extracellular matrix that is now considered the most common identifiable condition leading to the development of open-angle glaucoma, ${ }^{1}$ exfoliation glaucoma (XFG), and subsequent blindness ${ }^{1-3}$ XFG comprises over $50 \%$ of openangle glaucomas in some countries ${ }^{2,4-8}$ and its socioeconomic importance has increased considerably in recent years. ${ }^{1-3}$ At presentation XFG varies from primary open-angle glaucoma (POAG) due to older age, ${ }^{5,7,9}$ worse Intraocular pressure (IOP) characteristics, ${ }^{10-12}$ lower visual acuity, ${ }^{2,8}$ more visual field damage, ${ }^{13,14}$ and heavier trabecular pigmentation. ${ }^{8,14}$ XFG subjects often present with unilateral glaucoma, ${ }^{5,6,15}$ exhibit a high mean IOP, ${ }^{10,12,14}$ a wider fluctuation of $24-\mathrm{h} \mathrm{IOP}, 10,11$ and the IOP level may be rising with progression of the disease. ${ }^{16}$ It is therefore more likely for adjunctive therapy for multiple drugs and maximum medical therapy to be employed in the medical treatment of XFG. ${ }^{17-24}$
${ }^{1}$ University

Department of Ophthalmology AHEPA Hospital

Thessaloniki Greece

${ }^{2}$ Private Practice Thessaloniki

Greece

${ }^{3}$ University

Department of

Ophthalmology

Larissa, Greece

${ }^{4}$ University Department of Ophthalmology

Alexandroupolis, Greece

${ }^{5}$ Papageorgiou General Hospital

Thessaloniki, Greece

${ }^{6}$ Prefectural Hospital of

Korinthos

Korinthos, Greece

${ }^{7}$ Pharmaceutical Research Network, LLC Charleston, SC, USA

${ }^{8}$ Carolina Eye Institute at the University of South Carolina School of Medicine Columbia, SC, USA

Correspondence: WC Stewart MD Pharmaceutical Research Network, LLC 1639 Tatum Street Charleston, SC 29412, USA Tel: + 8437626500 Fax: + 8437627444

E-mail:prnc@

bellsouth.net

Received: 15 April 2003 Accepted in revised form: 10 October 2003

Published online:

27 February 2004

Clinical sites were supported by a grant from Pharmacia Hellas, Greece.

Pharmaceutical Research Network, LLC received no clinical funding for this study. 
It is well documented that XFG has a worse prognosis than POAG, ${ }^{25-29}$ but it has never been elucidated to date whether this is due to the difference in age, the worse IOP characteristics of XFG, or to an often quoted 'poorer response' of XFG to medications. Although in the literature there is often a suggestion that XFG demonstrates a poorer response to medications, ${ }^{17,20,28}$ this belief stems from retrospective data on glaucoma cohorts with different baseline IOPs and relies on evidence from a time when only timolol and pilocarpine were available as treatment options for this glaucoma. Surprisingly, to date there is very limited information with regard to the response and success rate of XFG with the newer medications (brimonidine, latanoprost, etc.). Since glaucomatous damage occurs more rapidly in $\mathrm{XFG}^{2,14}$ and the rate of subsequent blindness is twice that seen with POAG, ${ }^{16,25,28}$ determining the most effective initial therapy and the best medical stepwise therapy in $\mathrm{XFG}$ is especially important to help control these subjects.

Latanoprost is currently one of the most popular choices in glaucoma treatment worldwide. ${ }^{30-35}$ It is thus important to determine the success of monotherapy with latanoprost in XFG and compare the diurnal control obtained with this medication vs that with a classic drug of choice, timolol maleate, to facilitate the future management of this important secondary glaucoma.

\section{Materials and methods}

\section{Subjects}

Subjects were enrolled in this multicentre study if they had a clinical diagnosis of XFG, or ocular hypertension and XFS in at least one eye (study eye). If both eyes were eligible one eye was chosen at random. At screening, the IOP had to be controllable on monotherapy in the study eye and could safely undergo washout from their current medication in such a way that clinical stability of vision and the optic nerve is assured throughout this trial. All subjects were 39 years of age or older and prior to enrollment signed an informed consent form. At baseline, the mean untreated IOP (two measurements within one hour at the 1000 hour baseline time point) had to be between $22-36 \mathrm{mmHg}$ inclusive. All subjects had open angles consistent in appearance with XFG. In the contralateral eyes, not included in the study, the IOP had to be controllable on no drug therapy, on the study medicine, or with laser/surgery. In the study eye, visual acuity had to be 0.3 on the Snellen chart or better.

Subjects were excluded if they had any abnormality preventing reliable applanation tonometry in the study eye; concurrent infectious/noninfectious conjunctivitis; keratitis or uveitis in either eye; any history of allergic hypersensitivity or poor tolerance to any components of the medications used in this trial; intraocular conventional surgery or laser surgery in the study eye; and according to the investigator's best judgement, risk of visual field or visual acuity worsening as a consequence of participation in the trial. Subjects were also excluded if they exhibited progressive retinal or optic nerve disease apart from glaucoma; if they were females of childbearing potential not using reliable means of birth control; pregnant or lactating females; and had any clinically significant, serious, or severe medical or psychiatric condition. Individuals who did not wish to risk darkened irides or eyelash changes during the trial were also not enrolled as were not individuals with active or potential ocular inflammation (including cystoid macula oedema), history of ocular herpes simplex or macular oedema, any contraindication to $\beta$ adrenergic blockers, including reactive airway disease, decompensated heart failure, second or third-degree heart block, or sick sinus syndrome. Subjects using systemic $\beta$-blockers were not enrolled in this study.

\section{Procedures}

All subjects signed an Institutional Review Boardapproved informed consent form before entering this study. At the screening exam (Visit 1, Day -30), and all subsequent exams, IOPs were measured with a recently calibrated Goldmann applanation tonometer, visual acuity was measured on a Snellen visual acuity chart, and slit-lamp biomicroscopy as well as stereoscopic evaluation of the fundi were performed. Visual field assessment was performed at Visit 1 on the Humphrey Field Analyzer (program 24-2) or Octopus (G1 program). If subjects were treated prior to enrollment, they discontinued their medication according to a standard timetable of washing out (4 weeks for prostaglandin analogues and $\beta$-blockers, etc.).

Subjects returned to the clinic after the washout period for their untreated diurnal assessment (performed at 0800, 1000, 1400, and 2000) (Visit 2, Day 0). They were then randomized to the appropriate medication, either timolol maleate $0.5 \%$ (Vianex/MSD, Athens, Greece) one drop dosed at 0800 and 2000 hours, or latanoprost $0.005 \%$ (Pharmacia Hellas, Athens, Greece) dosed at 2000 hour and placebo (placebo artificial tears, supplied by the courtesy of Pharmacia Hellas) dosed at 0800 hours. Subjects returned for safety visits at 2 and 6 weeks (Visits 3 and 4). They returned at 3 months for a treated diurnal assessment of their IOP (Visit 5). During the diurnal IOP measurements at 3 months after initiation of therapy, the IOP was first measured at 0800 hours prior to instillation of the morning drop (ie, evaluation of the trough IOP), 
$2 \mathrm{~h}$ after instillation of the morning medication (peak IOP), at 1400 hours (afternoon IOP), and at 2000 hours (trough IOP for both drugs). At all time points the allowable range was $\pm 1 \mathrm{~h}$.

The medicine bottles were not masked. However, the physicians who performed the ocular examination, and those who obtained the patient history information, were not aware of the patient's treatment regimen. Further, the same physician recorded the patient's adverse event history for each visit. In addition, the same physician (but different from the physician who inquired about adverse events) measured the IOP at each visit.

At Visits 3-5, subjects were queried as to whether or not they had experienced symptoms suggesting an adverse event and about their general compliance to the masked medication dosing regimen since their last scheduled visit. Only compliant subjects were continued in the trial. Subjects were dismissed from the trial at the end of the treated diurnal assessment unless an unresolved adverse event existed.

\section{Statistics}

The statistics were analysed by Pharmaceutical Research Network, LLC (Charleston, SC, USA). All data analyses were two sided and a $0.05 \alpha$ level was used. Both the reduction and absolute levels of IOPs between treatment groups were analyzed by an unpaired t-test for intergroup analysis. This study provided an $80 \%$ power that a difference of $2.0 \mathrm{mmHg}$ could be excluded between the IOP of timolol maleate and latanoprost. A standard deviation of $3.5 \mathrm{mmHg}$ was assumed. The mean deviation was also analysed by an unpaired $t$-test. A Bonferroni correction $(\alpha / 5)$ was used to correct the $P$-values for multiple comparisons separately for the absolute IOP level and the reduction in pressure from baseline.

Safety parameters for ranked intergroup analysis were evaluated with a Mann-Whitney $U$ and unranked were evaluated by a $\chi^{2}$ test as appropriate. Adverse events were evaluated with a $\chi^{2}$ or Fisher's Exact test as appropriate.

\section{Results}

\section{Subjects}

A total of 109 consecutive subjects were prospectively enrolled in six centres around Greece during 1 year. Out of these, 103 subjects $(94.5 \%)$ completed the study. Of the six subjects who discontinued, two were lost to followup, one had poor IOP control on timolol, and three were discontinued due to adverse events. All subjects were Caucasian. There was no difference with regard to age,
Table 1 Subject characteristics

\begin{tabular}{lccc}
\hline Demographics & Latanoprost, $n=51$ & Timolol, $n=52$ & P-value \\
\hline Age (years) & $69.3 \pm 7.9$ & $70.2 \pm 6.9$ & 0.54 \\
Female & 29 & 28 & \\
Male & 22 & 24 & \\
XFG $^{\text {a }}$ & 35 & 38 & \\
XFS $^{\mathrm{b}}$ & 16 & 14 & \\
Visual acuity $^{\mathrm{c}}$ & $0.71 \pm 0.24$ & $0.69 \pm 0.23$ & 0.68 \\
Cup/disc ratio $_{\text {Visual field (dB) }}^{\text {d }}$ & $0.6 \pm 0.2$ & $0.6 \pm 0.1$ & 0.38 \\
Vin $^{2}$ & $7.11 \pm 4.48$ & $7.45 \pm 5.52$ & 0.73 \\
\hline
\end{tabular}

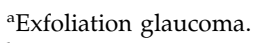

${ }^{b}$ Exfoliation syndrome.

'Taken as a ratio of Snellen visual acuity (ie, $\frac{6}{12=0.5}$ ).

${ }^{\mathrm{d}}$ Mean deviation.

gender, baseline IOP, visual acuity, mean visual field defect in subjects with XFG, or mean cup-to-disc ratio between treatment groups (see Table 1). In total, 51 subjects were randomized to receive latanoprost $0.005 \%$ and 52 were randomized to receive timolol maleate $0.5 \%$.

\section{Intraocular pressure}

Table 2 and Figure 1 demonstrate the IOP results with the two regimens. The baseline diurnal-untreated IOP for the latanoprost group was $24.9 \pm 3.2 \mathrm{mmHg}$, whereas the baseline diurnal-untreated IOP for the timolol group was $24.7 \pm 2.8 \mathrm{mmHg}$. Both treatments significantly reduced the baseline IOP at each time point and for the diurnal curve following 3 months of chronic dosing $(P<0.0001)$ (Table 2). Latanoprost, however, had a significantly lower mean IOP at 0800 hours $(P=0.0002)$. However, the IOPs were not statistically different at the 1000 hours $(P=0.37), 1400$ hours $(P=0.03)$, and 2000 hours $(P=0.71)$ time points and for the mean diurnal curve $(P=0.07)$ after the Bonferroni correction.

Likewise, latanoprost treatment reduced the IOP from baseline significantly more than timolol at $0800 \mathrm{~h}$ $(P<0.0001)$ (12 h following dosing) following three months of chronic dosing. Timolol reduced the IOP just as well as latanoprost at 1000, 1400, and 2000 hours (2, 8, and $12 \mathrm{~h}$ following dosing) and for the diurnal curve $(P=0.03)$ after the Bonferroni correction.

Latanoprost treatment obtained a significantly better diurnal range (ie, smaller fluctuation of treated IOP) compared with timolol $(2.4 \pm 1.2$ vs $3.2 \pm 1.5 \mathrm{mmHg}$, $P=0.0017)$ and a better mean maximum IOP $(P=0.03)$.

\section{Safety}

Table 3 shows the ocular adverse effects for both medications and Table 4 shows the systemic adverse events. There was significantly greater conjunctival hyperaemia with latanoprost $(P=0.01)$. One subject was 
Table 2 IOP reduction ( $\mathrm{mm} \mathrm{Hg} \pm$ standard deviation)

\begin{tabular}{|c|c|c|c|c|c|}
\hline & Baseline latanoprost & Latanoprost & Baseline timolol & Timolol & P-value between groups \\
\hline \multicolumn{6}{|l|}{ IOP reduction } \\
\hline 0800 hours & $26.0 \pm 3.4$ & $17.5 \pm 3.1$ & $25.5 \pm 3.1$ & $19.5 \pm 2.2$ & 0.0002 \\
\hline 1000 hours & $26.5 \pm 3.8$ & $17.9 \pm 3.3$ & $26.4 \pm 3.2$ & $18.4 \pm 2.3$ & 0.37 \\
\hline 1400 hours & $24.2 \pm 3.4$ & $16.7 \pm 3.0$ & $23.9 \pm 3.0$ & $17.9 \pm 2.2$ & 0.03 \\
\hline 2000 hours & $23.2 \pm 3.1$ & $17.5 \pm 2.9$ & $22.8 \pm 3.1$ & $17.3 \pm 2.1$ & 0.71 \\
\hline Diurnal & $24.9 \pm 3.2$ & $17.4 \pm 2.9$ & $24.7 \pm 2.8$ & $18.3 \pm 1.9$ & 0.07 \\
\hline Maximum & $27.1 \pm 4.0$ & $18.6 \pm 3.4$ & $27.0 \pm 3.2$ & $19.9 \pm 2.2$ & 0.03 \\
\hline Minimum & $22.5 \pm 2.6$ & $16.3 \pm 2.7$ & $22.3 \pm 2.8$ & $16.7 \pm 1.8$ & 0.35 \\
\hline Range & $4.6 \pm 1.9$ & $2.3 \pm 1.2$ & $4.7 \pm 1.7$ & $3.2 \pm 1.5$ & 0.0017 \\
\hline \multicolumn{6}{|c|}{ Baseline treatments } \\
\hline 0800 hours & & $8.5 \pm 2.9$ & & $6.0 \pm 2.8$ & $<0.0001$ \\
\hline 1000 hours & & $8.6 \pm 3.2$ & & $8.1 \pm 3.0$ & 0.38 \\
\hline 1400 hours & & $7.4 \pm 3.1$ & & $6.0 \pm 3.1$ & 0.02 \\
\hline 2000 hours & & $5.6 \pm 2.8$ & & $5.5 \pm 3.2$ & 0.76 \\
\hline Diurnal & & $7.5 \pm 2.7$ & & $6.4 \pm 2.8$ & 0.03 \\
\hline
\end{tabular}

${ }^{*} P$-value for latanoprost and timolol separately at all points was $<0.0001$, latanoprost showed a significantly greater IOP reduction at 0800 hours after a Bonferroni correction.

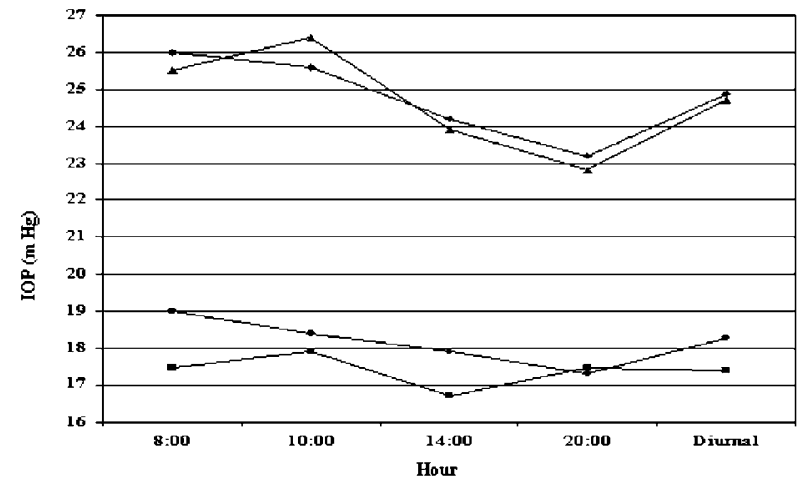

Figure 1 Diurnal mean IOPs for latanoprost at baseline (diamonds) and with latanoprost treatment (squares) and timolol at baseline (triangles) and with timolol treatment (circles).

withdrawn from the latanoprost group due to ocular intolerance, and one subject dropped out due to the occurrence of floaters in his study eye. In the timolol group, one subject was withdrawn due to bradycardia.

A number of systemic side effects were recorded in the study (Table 4). However, there were no differences between groups for any individual adverse event.

\section{Discussion}

The approach to the management of the subject with XFG may not be similar to primary POAG and the response to the new medications may differ when compared to POAG. ${ }^{3,11,19}$ Often the initial response to medications is greater with XFG, but in the long term these subjects can be more difficult to control with medical therapy than those with POAG. ${ }^{14,17,24}$ It is uncertain whether this is
Table 3 Ocular side effects (number of events)

\begin{tabular}{lccc}
\hline Side effect & Latanoprost & Timolol & P-value \\
\hline Conjunctival hyperemia & 8 & 1 & 0.01 \\
Corneal SPK $^{\mathrm{a}}$ & 3 & 5 & 0.48 \\
Stinging & 3 & 4 & 0.72 \\
Dry eye sensation & 2 & 4 & 0.41 \\
Itchiness & 4 & 2 & 0.39 \\
Foreign body sensation & 3 & 3 & 0.98 \\
Burning on instillation & 2 & 2 & 0.98 \\
Hypertrichosis & 3 & 0 & 0.08 \\
Lid erythema & 2 & 0 & 0.15 \\
Hyperchromia of the iris & 2 & 0 & 0.15 \\
Posterior vitreous detachment & 0 & 1 & 0.31 \\
Blurring of near vision & 1 & 0 & 0.31 \\
\hline
\end{tabular}

${ }^{\text {aSPK }}=$ superficial punctate epitheliopathy.

Table 4 Systemic side effect (number of events)

\begin{tabular}{lccc}
\hline Side Effect & Latanoprost & Timolol & P-value \\
\hline Increased dyspnoea & 0 & 2 & 0.16 \\
Exacerbation of insomnia & 0 & 1 & 0.32 \\
Increased fatigue & 0 & 1 & 0.32 \\
Headaches & 1 & 0 & 0.32 \\
Upper respiratory tract infection & 1 & 0 & 0.32 \\
Hypoglycemia & 1 & 0 & 0.32 \\
Elevated systemic blood pressure & 1 & 0 & 0.32 \\
\hline
\end{tabular}

due to the declining efficacy of the medications themselves, ${ }^{2,16,20}$ the significantly higher mean IOP values, ${ }^{17}$ the greater fluctuation of $24-\mathrm{h} \mathrm{IOP},{ }^{23,24}$ or the rising IOP during the course of XFG. ${ }^{16}$ In the past, XFG required surgical intervention more often and had a worse prognosis despite recourse to surgery earlier and more often. ${ }^{2,3,9,12,22,25}$ However, these observations were 
made when medical therapy comprised mainly $\beta$-blockers, pilocarpine, and epinephrine. The impact of the newer medications on the success of medical therapy in XFG has yet to be taken into consideration.

To date, the specific target diurnal IOP for XFG has not yet been addressed. It is conceivable that the target IOP is different in XFG and it is possible that XFG, being a hypertensive glaucoma, may respond better to long-term IOP reduction compared with POAG. In a recent study, examining the role of IOP reduction in XFG progression, we documented a clear-cut benefit for IOP reduction in many subjects with XFG, with only $28 \%$ of XFG subjects deteriorating with a mean IOP lower than $17 \mathrm{mmHg}$ over more than 5 years of follow-up. ${ }^{36}$ Thus, it is important to investigate how successful timely medical or surgical IOP reduction can be obtained.

Latanoprost is one of the most successful antiglaucoma medications available today and is fast becoming the gold standard of glaucoma therapy in POAG. ${ }^{30,37}$ Unfortunately, to date there are limited data with respect to the efficacy of this medication in controlling the IOP in XFG. $^{38}$

In this current study, we investigated the diurnal IOP reduction at four time points covering a period of 12 daytime hours to help establish the potency of these two medications in XFG. Both treatments significantly reduced the diurnal baseline IOP. Following a Bonferroni correction, the absolute IOP levels and the reduction in pressure from the nontreated baseline showed a nonstatistical trend in favour of latanoprost. The lack of significance may have resulted from, at least in part, the fact that the study was slightly underpowered (80\% power to detect a $2.0 \mathrm{mmHg}$ difference). Sponsored clinical trials typically are powered statistically to detect a $1.5 \mathrm{mmHg}$ difference.

Latanoprost treatment, however, demonstrated a significantly better diurnal range and better mean peak IOP compared with timolol. The smaller fluctuation of treated IOP may confer a long-term benefit in the management of XFG since it has been shown in POAG that the less the fluctuation, the better the prognosis in the management of glaucoma. ${ }^{39-41}$

With regard to specific time points, latanoprost treatment reduced the IOP significantly more than timolol and provided a lower absolute IOP at 0800 hours (12 $\mathrm{h}$ following dosing). However, only a trend of a lower IOP existed (after the Bonferroni Correction) with latanoprost for the other time points and the diurnal curve following 3 months of chronic dosing. It is not surprising that latanoprost is more efficacious than timolol at 0800 hours since this is the trough point for timolol, but probably the peak efficacy for latanoprost. It has been shown that latanoprost works better $12-24 \mathrm{~h}$ after administration. ${ }^{35}$ Considering the proximity of our diurnal baseline IOP time points to that of the latanoprost regulatory studies, our results indicate a similar, but slightly less, IOP effect in comparison with that found for POAG in these previously published multicentre studies. $^{31,32,34}$

With regard to diurnal efficacy, our study shows that both latanoprost and timolol appear to be at least as efficacious in XFG as previously reported to be in POAG. ${ }^{31,32}$ The specific response of the various medications with XFG has been a topic of controversy., Even with timolol maleate, the gold standard against which medications are measured, eyes with XFG have been reported to respond in a varied way in comparison with POAG. Aasved et al. ${ }^{42}$ have reported a worse response, whereas others suggest an equal, ${ }^{43}$ or greater ${ }^{11}$ ocular hypotensive effect. Konstas et al. ${ }^{11}$ evaluated the 24-h response with timolol $0.5 \%$ and showed that despite a greater initial IOP reduction in the XFG subjects, they still had a higher mean IOP and greater fluctuation in the 24-h IOP curve than subjects with POAG. Little previous information regarding the diurnal efficacy of latanoprost in EXG is available. Konstas et al. ${ }^{24}$ demonstrated a similar diurnal IOP response with adding latanoprost, as a third-line medication, to timolol and dorzolamide compared to pilocarpine $4 \%$.

To date, it is not known if latanoprost, by diverting aqueous to the uveoscleral outflow, has more or less of a beneficial effect on XFG vs medications that reduce aqueous production like timolol or vis-à-vis POAG. Furthermore, it has not been elucidated if the pigment granules liberated in XFG are deposited in the uveoscleral outflow, and if so, whether this plays a role in the context of XFG.

This study suggests that latanoprost provides a statistically lower 0800 hours IOP with similar safety than timolol as monotherapy in the treatment of XFG. The favourable diurnal IOP response to latanoprost compared to timolol maleate in XFG would be useful in guiding ophthalmologists in the selection of first-line medication in the management of XFG. However, it should be noted that the present study is not a full 24-h study and thus nighttime IOPs have not been adequately assessed.

\section{References}

1 Ritch R. Exfoliation syndrome: the most common identifiable cause of open-angle glaucoma. J Glaucoma 1994; 3: $176-178$.

2 Ritch R, Schlötzer-Schrehardt U. Exfoliation syndrome. Surv Ophthalmol 2001; 45: 265-315, (Review). 
3 Ritch R, Schlötzer-Schrehardt U, Konstas AGP. Why is glaucoma associated with exfoliation syndrome? Prog Retin Eye Res 2003; 22: 253-275.

4 Vesti E, Kivel AT. Exfoliation syndrome and exfoliation glaucoma. Prog Retin Eye Res 2000; 19: 268-345.

5 Ringvold A. Epidemiology of the pseudo-exfoliation syndrome. Acta Ophthalmol Scand 1999; 77: 371-375.

6 Forsius H. Exfoliation syndrome in various ethnic populations. Acta Ophthalmol Suppl 1988; 184: 71-85.

7 Kozobolis VP, Papatzanaki M, Vlachonikolis IG, Pallikaris IG, Tsambarlakis IG. Epidemiology of pseudoexfoliation in the island of Crete (Greece). Acta Ophthalmol Scand 1997; 75: 726-729.

8 Konstas AGP. Morphological and clinical studies on the exfoliation syndrome and open angle glaucoma. $\mathrm{PhD}$ Thesis, Glasgow 1993, pp. 1-367.

9 Konstas AG, Stewart WC, Stroman GA, Sine CS. Clinical presentation and initial treatment patterns in patients with exfoliation glaucoma vs primary open-angle glaucoma. Ophthalmic Surg Lasers 1997; 28: 111-117.

10 Konstas AGP, Mantziris DA, Stewart WC. Diurnal intraocular pressure in untreated exfoliation and primary open-angle glaucoma. Arch Ophthalmol 1997; 115: 182-185.

11 Konstas AG, Mantziris DA, Cate EA, Stewart WC. Effect of timolol on the diurnal intraocular pressure in exfoliation and primary open-angle glaucoma. Arch Ophthalmol 1997; 115: 975-979.

12 Konstas AG, Jay JL, Marshall GE, Lee WR. Prevalence, diagnostic features and response to trabeculectomy in exfoliation glaucoma. Ophthalmology 1993; 100: 619-627.

13 Teus MA, Castejon MA, Calvo MA, Perez-Salaices P, Marcos A. Intraocular pressure as a risk factor for visual field loss in pseudoexfoliative and in primary open-angle glaucoma. Ophthalmology 1998; 105: 2225-2230.

14 Konstas AGP, Dimitracoulias N, Konstas PA. Exfoliation syndrome and open angle glaucoma. Klin Monatsbl Augenheilkd 1993; 202: 259-268.

15 Brooks AMV, Gillies WE. The presentation and prognosis of glaucoma in pseudoexfoliation of the lens capsule. Ophthalmology 1988; 95: 271-276.

16 Lindblom B, Thorburn W. Functional damage at diagnosis of primary open angle glaucoma. Acta Ophthalmol (Copenh) 1984; 62: 223-229.

17 Tarkkanen A. Treatment of chronic open-angle glaucoma associated with pseudoexfoliation. Acta Ophthalmol (Copenh) 1965; 43: 514-523.

18 Konstas AG, Tsatsos I, Kardasopoulos A, Bufidis T, Maskaleris G. Preoperative features of patients with exfoliation glaucoma and primary open-angle glaucoma. The AHEPA study. Acta Ophthalmol Scand 1998; 76: 208-212.

19 Konstas AG, Maltezos A, Mantziris DA, Sine CS, Stewart WC. The comparative ocular hypotensive effect of apraclonidine with timolol maleate in exfoliation $v s$ primary open-angle glaucoma patients. Eye 1999; 13 (Part 3a): 314-318.

20 Blika S, Saunte E. Timolol maleate in the treatment of glaucoma simplex and glaucoma capsulare. Acta Ophthalmol (Copenh) 1982; 60: 967-976.

21 Heijl A, Strahlman E, Sverrisson T, Brinchman-Hansen O, Puustjarvi T, Tipping R. A comparison of dorzolamide and timolol in patients with pseudoexfoliation and glaucoma or ocular hypertension. Ophthalmology 1997; 104: 137-142.

22 Moreno-Montanes J, Alvarez-Serna A, Alcolea-Paredes A. Pseudoexfoliative glaucoma in patients with open-angle glaucoma in the northwest of Spain. Acta Ophthalmol (Copenh) 1990; 68: 695-699.

23 Konstas AG, Maltezos A, Bufidis T, Hudgins AG, Stewart WC. Twenty-four hour control of intraocular pressure with dorzolamide and timolol maleate in exfoliation and primary open-angle glaucoma. Eye 2000; 14 (Part 1): 73-77.

24 Konstas AG, Lake S, Maltezos AC, Holmes KT, Stewart WC. 24 hintraocular pressure reduction with latanoprost compared to pilocarpine as third line therapy in exfoliation glaucoma. Eye 2001; 15 (Part 1): 59-63.

25 Thorburn W. The outcome of visual function in capsular glaucoma. Acta Ophthalmol 1988; 184 (Suppl): 132-137.

26 Aasved $\mathrm{H}$. The frequency of optic nerve damage and surgical treatment in chronic simple glaucoma and capsular glaucoma. Acta Ophthalmol (Copenh) 1971; 49: 589-600.

27 Lindblom B, Thorburn W. Prevalence of visual field defects due to capsular and simple glaucoma in Halsingland, Sweden. Acta Ophthalmol (Copenh) 1982; 60: 353-361.

28 Olivius E, Thorburn W. Prognosis of glaucoma simplex and glaucoma capsulare. A comparative study. Acta Ophthalmol (Copenh) 1978; 56: 921-934.

29 Pohjanpelto P. Long-term prognosis of visual field in glaucoma simplex and glaucoma capsulare. Acta Ophthalmol (Copenh) 1985; 63: 418-423.

30 Stjernschantz JW. From PGF(2alpha)-isopropyl ester to latanoprost: a review of the development of Xalatan: the Proctor Lecture. Invest Ophthalmol Vis Sci 2001; 42: 1134-1145.

31 Watson P, Stjernschantz J. A six-month, randomized, double-masked study comparing latanoprost with timolol in open-angle glaucoma and ocular hypertension. The Latanoprost Study Group. Ophthalmology 1996; 103: 126-137.

32 Alm A, Stjernschantz J. Effects on intraocular pressure and side effects of $0.005 \%$ latanoprost applied once daily, evening or morning. A comparison with timolol. Scandinavian Latanoprost Study Group. Ophthalmology 1995; 102: 1743-1752.

33 Alm A, Widengard I, Kjellgren D, Soderstrom M, Fristrom B, Heijl A et al. Latanoprost administered once daily caused a maintained reduction of intraocular pressure in glaucoma patients treated concomitantly with timolol. Br J Ophthalmol 1995; 79: 12-16.

34 Camras CB. Comparison of latanoprost and timolol in patients with ocular hypertension and glaucoma: a sixmonth masked, multicenter trial in the United States. The United States Latanoprost Study Group. Ophthalmology 1996; 103: 138-147.

35 Konstas AG, Maltezos AC, Gandi S, Hudgins AC, Stewart WC. Comparison of 24-hour intraocular pressure reduction with two dosing regimens of latanoprost and timolol maleate in patients with primary open-angle glaucoma. Am J Ophthalmol 1999; 128: 15-20.

36 Konstas AG, Hollo G, Astakhov YS, Teus MA, Akopov EL, Leech JN et al. Factors associated with long-term progression or stability in exfoliation glaucoma. Arch Ophthalmol 2004; 122: 29-33.

37 Hedman K, Watson PG, Alm A. The effect of latanoprost on intraocular pressure during 2 years of treatment. Surv Ophthalmol 2002; 47 (Suppl 1): S65-76. 
38 Konstas AG, Kozobolis VP, Tersis I, Leech J, Stewart WC The efficacy and safety of the timolol/dorzolamide fixed combination vs latanoprost in exfoliation glaucoma. Eye 2003; 17: 41-46.

39 Stewart WC, Chorak RP, Hunt HH, Sethuraman G. Factors associated with visual loss in patients with advanced glaucomatous changes in the optic nerve head. Am J Ophthalmol 1993; 116: 176-181.

40 Stewart WC, Kolker AE, Sharpe ED, Day DG, Holmes KT, Leech JN et al. Factors associated with long-term progression or stability in primary open-angle glaucoma. Am J Ophthalmol 2000; 130: 274-279.
41 Asrani S, Zeimer R, Wilensky J, Gieser D, Vitale S, Lindenmuth $\mathrm{K}$. Large diurnal fluctuations in intraocular pressure are an independent risk factor in patients with glaucoma. J Glaucoma 2000; 9: 134-142.

42 Aasved H, Seland JH, Slagsvold JE. Timolol maleate in treatment of open angle glaucoma. Acta Ophthalmol (Copenh) 1979; 57: 700-708.

43 Takki KK, Klemetti A, Valle O. The IOP-lowering effect of timolol in simple and capsular glaucoma. A multicenter study in Finland. Graefes Arch Clin Exp Ophthalmol 1982; 218: 83-87. 